\title{
ALGUNAS NOTAS SOBRE EL USO DE LOS ADVERBIOS EN MENTE EN EL HABLA INFANTIL*
}

\section{EI ADVERBIO: UNA GATEGORÍA ESPECIAL}

El adverbio es una categoría gramatical caracterizada por sus "límites difusos". En efecto, en cualquier estudio sobre el adverbio, sea cual sea su enfoque teórico, nos encontramos con diversas argumentaciones que ponen de manifiesto el carácter variado y complejo de su naturaleza formal, funcional y semántica y por ende, la dificultad de su definición y clasificación ${ }^{2}$. En este trabajo, pretendo sacar del "cajón de sastre"3 de los adverbios, a los terminados en mente $e^{4}$ y hacer una primera cala de su

* Como suele decirse en estos casos, la responsabilidad de esta nota es sólo mía. Sin embargo, agradezco a los profesores Luis Fernando Lara y Pedro Martín Butragueño por sus valiosos comentarios y sugerencias. Contribuyeron a enriquecerme y a enriquecer el trabajo.

${ }^{1}$ Hernanz y Brucart 1987, p. 33.

${ }^{2}$ El problema de la definición y clasificación del adverbio rebasa, con mucho, los objetivos de este trabajo. Por el momento, quedémonos con la cita de Ana María BarRenechea, como representativa de las variaciones sobre el tema: "el adverbio se presenta como una categoría híbrida que la gramática tradicional ha elaborado y cuyos problemas sintácticos y semánticos no han sido resueltos en forma satisfactoria" (1979, p. 313). Veánse también JESPERSEN 1975, p. 89; Alcina y Blecua 1975, pp. 700-701; Lázaro Mora 1987, p. 257; Hernanz y Brucart 1987, p. 268.

${ }^{3}$ Por su carácter multifacético, es un lugar común en la literatura llamar a los adverbios con este ilustrativo término de cajón de sastre: "la lingüística estructural, la gramática generativa y la pragmática lingüística, aliadas o no con la lógica formal, han tratado y están tratando de poner un poco de orden en ese cajón de sastre del adverbio" (GIRón Alconchel 1991, p. 7).

${ }^{4}$ Estos adverbios no escapan a los rasgos de su clase "su heterogeneidad no sólo afecta a aspectos semánticos y formales sino que se extiende al dominio de la sintaxis" (Hernanz y Brucart 1987, p. 268). Para un estudio completo y descriptivo de los adverbios en mente, véase EGEA 1979. 
uso en dos momentos concretos del habla infantil: seis y doce años, edades fundamentales pues representan los puntuales -inicio y término- de los años escolares. En efecto, a lo largo de estos años, el impacto de la escuela y los nuevos contextos comunicativos que supone, inciden directamente en el desarrollo lingüístico ${ }^{5}$. El niño va integrando, poco a poco, en el entramado discursivo, elementos - ya nuevos, ya viejos y reorganiza$\operatorname{dos}^{6}$ - sintácticos y semánticos que enriquecen su gramática y cohesionan su comunicación dentro de los nuevos marcos de interacción social en los que participa.

Hacia los seis años, "edad frontera", se da un paso sustantivo que va del qué se adquiere (estructuras y léxico, básicamente) de los primeros años de adquisición, al cómo (diversidad de situaciones comunicativas y de interlocutores) se adquiere de las etapas tardías (de siete a doce años). Si bien el niño de seis años ha adquirido la columna vertebral de su lengua, a lo largo de los años escolares tendrá que dar aún muchos y variados pasos para llegar a su dominio 7 .

De todos los aspectos de interés que presenta el uso de los adverbios en mente, dentro de este complejo proceso en el que se imbrican la adquisición-aprendizaje, aquí destacaré dos de los que, a mi parecer, son más relevantes. El primero es el concepto de modo, tiempo y cantidad que subyace a estos adverbios, nociones cuya dificultad se relaciona estrechamente con el desarrollo lingüístico y cognoscitivo del niño ${ }^{8}$.

Estos adverbios aunque pueden emerger muy temprano en el habla infantil en forma de simples items léxicos, no son domina-

${ }^{5}$ Cf. Romaine 1984, pp. 154-155. En su interesante estudio sobre el lenguaje de niños y adolescentes, esta autora destaca la importancia de las nuevas situaciones comunicativas a las que se enfrenta el niño durante sus años escolares. Véanse también Ellior 1983, Scott 1984, Karmiloff-Smith 1988, HOFFNER et al. 1990.

${ }^{6}$ La idea del desarrollo del lenguaje como un proceso de reorganización en el que las primeras formas adquiridas se transforman en nuevos contenidos, ha sido largamente trabajada en los estudios de adquisición del lenguaje. Cf. Slobin 1973, Bowerman 1984.

${ }^{7}$ Véanse Romaine 1984, Karmiloff-Smith 1988.

8 "The linguistic developments in adverbial connectivity undoubtedly have important cognitive and social analogues. Obviously, it is not coincidental that such structures begin to appear shortly after the onset of concrete operational thought with its attendant hallmarks in the ability to deal simultaneously with more than one concept, and to manipulate conceptual interrelationships mentally"' (ScotT 1984, p. 450). 
dos en toda su complejidad semántica por el niño. Con el desarrollo de otras habilidades - las discursivas, principalmente-, el niño incrementa el uso pertinente de estas categorías, en tanto que va desvelando el significado que les subyace con sus verdaderas implicaciones lógicas y semánticas ${ }^{9}$.

$\mathrm{El}$ otro aspecto importante de los adverbios en mente en relación con el habla infantil es su vinculación con la actitud y la intencionalidad ${ }^{10}$. Su uso relfeja la opción que tiene el hablante -el niño en este caso- de marcar su discurso con ciertos elementos que ponen de relieve su subjetividad ${ }^{11}$.

Desafortunadamente, hay una casi total ausencia de estudios de lenguaje infantil que profundicen en este importante tema de la subjetividad", que completa la visión total de las funciones del lenguaje en la producción infantil. A la par que el niño adquiere y aprende nuevas estructuras y significados, toma conciencia del valor de su propia actitud frente a su habla y de la carga emotiva y expresiva que puede imprimirle.

En suma, la producción de adverbios en mente en el habla infantil resulta importante porque supone un gradual dominio que atraviesa varios niveles: sintáctico, semántico y discursivo y que trasciende las fronteras de la mera competencia lingüística para llegar a la competencia comunicativa. Éste sería un hilo más para entender el entramado del desarrollo del lenguaje infantil.

${ }^{9}$ Hay una rica bibliografía en torno al problema del desarrollo y dominio de conceptos en el lenguaje infantil, pero sin duda, el punto de partida obligado en este caso son PIAGet 1981 y VygOtsky sía. Para la adquisición del inglés el tema se ha tratado ampliamente. Entre otros, Clark \& Clark 1977, De Villiers \& De Villiers 1978, Scott 1984, Elliot 1983, Hoffner et al. 1990 y Yousser 1990. En el español, en cambio, la investigación en esta área continúa siendo muy escasa: Peronard 1985, Barriga Villanueva 1990.

${ }^{10}$ Resulta interesante analizar la procedencia del morfema mente. Su formación proviene del sustantivo latino mens, mentis cuyo significado es mente, espiritu, intención (cf. Esbozo 1985, p. 200; las cursivas son mías).

${ }^{11}$ En su estudio sobre la enunciación, KERBRAT-ORECCHIONI afirma que la subjetividad del lenguaje es omnipresente pero que hay "lugares de anclaje" en los que se manifiesta más perceptiblemente la subjetividad: deícticos, adjetivos, verbos y adverbios en mente $(1987$, p. 44). En torno a la subjetividad del lenguaje, Benveniste dice: "es en y por el lenguaje como el hombre se constituye como sujeto; porque sólo el lenguaje funda en realidad, en su realidad que es la de ser, el concepto de "ego"" (1978, p. 180). Constituirse en sujeto de su lenguaje y hacerlo consciente es un proceso que necesariamente habrá de seguir un niño en su camino a la adquisición del lenguaje.

12 Cf. Barriga Villanueva 1990, pp. 182-239. 
UNA PRIMERA CALA DE LOS ADVERBIOS EN MENTE

Los adverbios que aquí analizo pertenecen al corpus de mi investigación doctoral ${ }^{13}$, que está formada por diversas conversaciones grabadas con veinticuatro niños, entre niños y niñas: doce de seis años y doce de doce años, de un estrato socioeconómico medio. Las grabaciones fueron hechas en dos sesiones de media hora cada una, con un intervalo aproximado de un mes entre una y otra. Durante estas grabaciones, dialogaba y conversaba con los niños sobre todo tipo de temas: experiencias personales, narraciones de cuentos, películas, telenovelas, relatos fantásticos, descripciones de juegos, discusiones de sucesos importantes en el momento, en fin, de todos aquellos temas que pudieran ser de interés para los niños y fomaran parte de su realidad cotidiana. Se buscaba, a través de esta situación dialógica (adulto-niño), obtener una producción, lo más espontánea y natural posible, que reflejara en las mismas condiciones la competencia de los niños —entendida ésta en un sentido amplio-, en dos momentos diferentes de su desarrollo lingüístico.

En general, los resultados del análisis del corpus fueron muy interesantes pues mostraron la realidad de este desarrollo, expresado, muy especialmente, en el nivel discursivo (narraciones, descripciones y diálogos). Como en toda investigación se abrieron muchos caminos nuevos. De ahí que ahora retome el de los adverbios como una rica veta en el conocimiento del lenguaje infantil.

Para tener una visión más clara y gráfica de los resultados de la producción de adverbios en mente en este corpus, veamos los resultados $^{14}$ reunidos en las tablas 1 y 2.

Como se desprende de estas tablas, los adverbios en mente son escasos en el habla de los niños de seis años, frente a una relativa abundancia en el habla de niños de doce (relación 1 a 10). Estos resultados no parecen fortuitos sino que obedecen a ciertos ras-

${ }^{13}$ El objetivo de esta investigación era comparar en una muestra transversal, la producción de algunas estructuras sintácticas, narraciones y algunos elementos lingüísticos, de los llamados subjetivos, con el fin primordial de analizar las diferencias que se dan en dos momentos fundamentales del desarrollo lingüístico (BaRRiga Villanueva 1990).

${ }^{14}$ En un estudio posterior, será interesante analizar si este uso de los adverbios en mente es general en los niños mayores, es un estilo personal o es más bien un uso motivado por el tipo de discurso. Recuérdese que lo que prevalecía en este corpus eran los diálogos y las narraciones. 
TABLA 1

\begin{tabular}{lll}
\hline \multicolumn{1}{c}{ Adverbios en "mente" } & 6 años & 12 años \\
\hline 1. aproximadamente & & 1 \\
2. constantemente & & 2 \\
3. desafortunadamente & & 1 \\
4. desgraciadamente & & 1 \\
5. difícilmente & 1 & 2 \\
6. directamente & & 3 \\
7. exactamente & & 4 \\
8. inclinadamente & & 1 \\
9. ingenuamente & 1 & 2 \\
10. inmediatamente & & 1 \\
11. lógicamente & & 1 \\
12. nuevamente & & 2 \\
13. posiblemente & & 2 \\
14. precisamente & & 1 \\
15. principalmente & & 1 \\
16. rápidamente & & 1 \\
17. realmente & 1 & 13 \\
18. sencillamente & & 4 \\
19. simplemente & & 5 \\
20. solamente & & 2 \\
21. sucesivamente & & 2 \\
22. últimamente &
\end{tabular}

TABLA 2

\begin{tabular}{lcc}
\hline Adverbios en "mente" & 6 años & 12 años \\
\hline Total de adverbios & 3 & 22 \\
Total de ocurrencias & 3 & 54 \\
Total de palabras producidas & 46020 & 81911 \\
$\%$ de adverbios & 0.0065 & 0.0659 \\
Relación & $1:$ & 10.14 \\
\hline
\end{tabular}

gos del habla vinculados directamente tanto con el desarrollo cognoscitivo y lingüístico, así como con el conocimiento del mundo de los niños en momentos clave de su formación. Como ya se había mencionado, los conceptos de modalidad, espacialidad, temporalidad o cantidad que subyacen a estos adverbios, resultan difíciles de comprender sobre todo para niños que inician los años escolares. Sin embargo, surge aún una duda: ¿es el adverbio en mente una categoría de adquisición tardía y de ahí su escasez a los seis años o se trata más bien de una producción restringida por el tipo de situación comunicativa no dominada aún por los más pequeños? 
Pero vayamos a la producción de los niños. Los adverbios en mente encontrados en el habla de seis años son nuevamente (de tiempo), solamente y exactamente (de modo $)^{15}$.

a) I: - ¿Y qué haces durante el simulacro?

$\mathrm{N}$ :- Ahí nos quedamos hasta que pase el temblor y nos metemos nuevamente a nuestro salón.

b) I: - ¿Y cuántos años tiene Marian?

$\mathrm{N}$ : - Tiene exactamente seis años pero yo le creí y me dijo que siete.

c) I: - ¿Y tú crees en los dragones?

$\mathrm{N}$ : - No pueden existir los dragones o que ya haigan solamente inventado esa historia hace muchos años.

Lo interesante de estos adverbios es que, aunque escasos, apuntan ya hacia una mayor conciencia del niño con respecto al contenido de su discurso ${ }^{16}$. Hay una subjetividad más involucrada que se manifiesta en el cuidado del niño por precisar su información, de objetivizarla, en el sentido de hacerla más cercana a su interlocutor ${ }^{17}$.

En los niños de doce años este uso se hace cada vez más presente en su discurso. De hecho, puede decirse que con los adverbios en mente recorren toda la gama de posibilidades expresivas

${ }^{15}$ Por el momento, no me detendré en las relaciones sintácticas que establecen estos adverbios con todos los elementos oracionales, pues supondría un análisis diferente del que aquí me he propuesto; sin embargo, es importante señalar la posición que ocupan los adverbios en la oración (en este caso pospuesta e intercalada) ya que ésta puede cambiar su significado. Esto en términos de adquisición, dominio y comprensión de la lengua es sumamente significativo. Lo importante para nosotros, por ahora, es subrayar la incipiente aparición de estos adverbios, si tenemos en cuenta además, que una estrategia común en el habla de los seis años es llenar el discurso con otros elementos de la subjetividad más orientados hacia la afectividad y la emotividad. De ahí la frecuencia de adverbios con valor intensificador: canta bien bonito, crecieron mucho mucho del mismo tamaño, estuvo muy bonito, muy bonito, muy padre, me gustó.

16 Ana María Barrenechea llama a algunos de estos adverbios en mente "operadores pragmáticos de actitud oracional" que indican la actitud del hablante, y distingue dos grandes clases cada una con sus propias expresiones: actitud emocional y graduación en el discurso aseverativo (1979, p. 317). Kerbrat-Orecchioni, por su parte, dice que estos adverbios son "modalizadores que implican «juicios de verdad" o «juicios sobre la realidad" y que especifican las condiciones y la naturaleza del hecho de habla" (1987, p. 155).

${ }^{17}$ En términos piagetianos, éste sería el paso de la etapa preoperatoria a la operatoria, en la que el niño, además de salir de su egocentrismo entra en una nueva fase de operaciones cognoscitivas (PIAGET 1981, pp. 61, 153). 
y valorativas que conllevan estos adverbios. Parten de los matices de frecuencia temporal, definida o indefinida, alta o baja ${ }^{18}$, como en:

a) Pues últimamente nada más fui una vez al cine porque como nosotros tenemos video y ya casi no vamos.

b) Se van hundiendo, cayendo constantemente de la lancha y se mueren.

c) Tuvimos muchos problemas porque no había llegado el doctor ni las enfermeras y así sucesivamente.

Los niños de doce años atraviesan también los terrenos resbaladizos de lo probable ${ }^{19}$, lo posible y lo real:

a) Difícilmente pude leer ese libro, era muy aburrido.

b) Ese viaje posiblemente lo haga con mi papá y con más maestros.

c) La maestra Luchita, bien amable me decía: "¿qué, no le entiendes?" y me explicaba, por eso, o sea, realmente mi maestra era ella.

d) La imaginación, cómo te diré, cosas que no existen, que el hombre cree haber vivido pero realmente no fueron.

De este grupo de ejemplos, centro mi atención en el $c$ y el $d$ puesto que presentan dos matices en el uso de realmente. En el $c$ el niño pondera las cualidades de la maestra para enfatizar su juicio y avalar su afirmación. En tanto que en $d$ la niña hace toda una explicación lógica para concluir que la imaginación tiene que ver con cosas que no existen. Estos procesos complejos y sutiles apoyan la idea de una madurez lingüística y cognoscitiva en vías de consolidación.

Siguiendo por la misma línea de la sutileza y los matices, detengámonos en este otro ejemplo:

a) Estaban criticando al gobierno, o sea, no directamente, pero sí más o menos fuerte.

18 Cf. Lázaro Mora 1987, pp. 260-262.

19 Los grados de probabilidad y certeza expresados en el habla infantil son de aparición tardía. HoffNer et al. hacen un estudio con éstos y concluyen que "children do not have the cognitive structures needed to distinguish between certainty and possibility until age seven, and the ability to compare degrees of probability does not develop until adolescence" (1990, p. 219). Véanse también Scott 1984 y Youssef 1990. 
Aquí la niña esconde el sentido literal del adverbio al acompañarlo de una negación para significar finalmente lo que ella quiere decir.

Entre todos los adverbios usados por los niños de doce años destaca la frecuente ocurrencia de solamente (por cierto, fue uno de los tres producidos por niños de seis años), lo encuentro como una especie de muletilla con énfasis en el valor modal:

a) Las chicas solamente quieren divertirse.

Como se ve, éste es un apoyo leve, frente a la fuerza y seguridad que pueden brindar otros adverbios también usados por estos niños de doce años, como en:

a) $\mathrm{Y}$ nos fuimos a un baile hawaiano, el hawaiano lógicamente.

b) Creer, existir, no, precisamente reencarnación no.

Cerremos el recorrido con aquellos adverbios que enfatizan los rasgos emotivos de la acción:

a) No yo no bailé, desafortunadamente no pude porque no tenía patines.

b) ...entonces mi tío murió, desgraciadamente, y ya no pudimos ir.

Nótese que estos adverbios situados en el vértice de lo emocional y en lo afectivo están alejados, desde el punto de vista de su significado, del frecuente uso que hacen los niños de elementos subjetivos que enfatizan también la emotividad. La diferencia está marcada, por la intención y el grado de conciencia lingüística ${ }^{20}$.

Hasta aquí los adverbios en mente más interesantes encontrados en mi estudio. Considero que esta primera cala ha mostrado el porqué de la necesidad de seguir abundando en estas paradójicamente difusas e iluminadoras piezas en el complejo andamiaje del desarrollo lingüístico infantil. 


\section{BIBLIOGRAFÍA}

Alcina Franch, Juan, y José Manuel Blecua 1975. Gramática española. Ariel, Barcelona.

Barrenechea, Ana María 1979. "Operadores pragmáticos de actitud oracional: los adverbios en mente", en Juan M. Lope Blanch (ed.), Estudios sobre el español hablado en las principales ciudades de América. UNAM, México, pp. 311-332.

Barriga Villanueva, Rebeca 1990. Entre lo sintáctico y lo discursivo. Un análisis comparativo del habla infantil. El Colegio de México, México (tesis doctoral inédita).

Benveniste, Emile 1978. Problemas de lingüistica general I. Trad. J. Almela. Siglo XXI, México.

BOWERMAM MELSSA 1984. Reorganizational processes in lexical and syntactic development" "en ERIo WANMER \& LILA R. GLITMAN (edso), Language acquistion. the state of an. Cambridge Unversity press, Cambridge-London, pp. 285-305.

Clark, Herbert H., \& Eve V. Clark 1977. Psychology and language. An introduction io psycholinguistics. Harcourt Brace, New York.

De Villiers, Jill G., \& Peter A. De Villiers 1978. Language acquisition. Harvard University Press, Cambridge, MA-London.

EGEA, E. R. 1979. Los adverbios terminados en "mente" en el español contemporáneo. Instituto Caro y Cuervo, Bogotá.

Elliot, Alison J. 1983. Child language. Cambridge University Press, Cambridge.

Girón Alconchel, José Luis 1991. Tiempo, modalidad y adverbio. (Significado y formación del adverbio "ya"). Universidad de Salamanca, Salamanca.

Hernanz, Maria liuisa, y José Maria Brucart 1987. La siniaxis. 1. Principios teóricos. La oración simple. Crítica, Barcelona.

Hoffner, Chntmia, Joanne Cantor, \& Diane M. Badzinski 1990. "Chil" dren's understanding of adverbs denoting degree of likelihood", JChL, $17,217-231$.

Jespersen, Otto 1975. La filosofía de la gramática. Trad. C. Manzano. Anagrama, Barcelona.

Kerbrat-Orecchoni, Catherine 1987. La enunciación. De la subjetividad en el lenguaje. Trads. G. Anfora y E. Gregores. Hachette, Buenos Aires.

Karmiloff-Smith, Annette 1988. "Some fundamental aspects of language development after age 5", en Paul Fletcher \& Michael Garman (eds.), Language acquisition. Studies in first language development. Cambridge University Press, Cambridge, pp. 455-474.

Lázaro Mora, Fernando A. 1987. "Sobre adverbios de tiempo", LEA, 9, 257-265.

Peronard, Marianne 1985. "Spanish prepositions introducing adverbial constructions", JChL, 12, 95-108.

Piaget, Jean 1981. Seis estudios de psicología. Trad. N. Petit. Seix Barral, Barcelona [la. ed. en francés: PUF, Paris, 1946].

Real Academia Española 1985. Esbozo de una nueva gramática de la lengua española. Espasa-Calpe, Madrid.

Romaine, Suzanne 1984. The language of children and adolescents. The acquisition 
of communicative competence. Basil Blackwell, New York. (Language in Society, 7).

SCOTT, CHERyl M. 1984. "Adverbial connectivity in conversations of children", JChL, 11, 423-452.

SLOBIN, DAN IsSAC 1973. Studies of child language development. Holt, Rinehart and Winston, New York.

Stubbs, Michael 1983. Discursive analysis. The sociolinguistic analysis of natural language. University of Chicago Press-Basil Blackwell, Chicago-Oxford.

Vygotsky, Lev S. 1973. Pensamiento y lenguaje. Teoría del desarrollo cultural de las funciones psiquicas. Pról. J. Itzigsohn. Alfa y Omega, México, s.a. [1 ${ }^{\text {a }}$ ed. en ruso: 1934; la. ed. en inglés: 1962].

YousSEF, VALERIE 1990. "The early development of perfect aspect: adverbial, verbal and contextual specification", $J C h L, 17,295-312$. 